\title{
RAYLEIGH WAVES IN A THERMOELASTIC GRANULAR MEDIUM UNDER INITIAL STRESS
}

\author{
S. M. AHMED
}

(Received 23 December 1998)

\begin{abstract}
We study the effect of initial stress on the propagation of Rayleigh waves in a granular medium under incremental thermal stresses. We also obtain the frequency equation, in the form of a twelfth-order determinantal expression, which is in agreement with the corresponding classical results.
\end{abstract}

Keywords and phrases. Rayleigh waves, thermoelastic waves, granular medium.

2000 Mathematics Subject Classification. Primary 74J15.

1. Introduction. The propagation of thermoelastic waves in a granular medium under initial stress has some applications in soil mechanics, earthquake science, geophysics, mining engineering, etc. The theoretical outline of the development of the subject from the mid-thirties was given by Paria [9]. The present paper, however, is based on the dynamics of granular media as propounded by Oshima $[7,8]$.

The medium under consideration is discontinuous such as one composed numerous large or small grains. Unlike a continuous body, each element or grain cannot only translate but also rotate about its centre of gravity. This motion is the characteristics of the medium and has an important effect upon the equations of motion to produce internal friction. It is assumed that the medium contains so many grains that they will never be separated from each other during the deformation and that the grain has perfect elasticity. The frequency equation of Rayleigh waves in a granular layer over a granular half-space was given by Bhattacharyya [2]. In [4], Elnaggar investigated the influence of initial stress of the waves propagation in a thermoelastic granular infinite cylinder. Recently [1], Ahmed discussed the influence of gravity on the propagation of waves in granular medium.

This paper is devoted to the study of the effect of initial stress on the propagation of Rayleigh waves in a granular medium under incremental thermal stresses. The medium under consideration is granular half-space overlain by a different granular layer and initial stresses present in this medium have considerable effect in the propagation of Rayleigh waves [3]. The wave velocity equation has been derived in the form of twelfth-order determinant. The roots of this equation are in general complex and the imaginary part of an appropriate root measures the attenuation of the waves. It is shown that the frequency of Rayleigh waves contains terms involving thermal coefficients and other terms involving initial stress and so the phase velocity changes with respect to this thermal coefficients and the initial stress. When there is no coupling between the temperature and the strain field in the absence of the initial stress, 
the derived frequency equation reduces to an equation in the form of ninth-order determinant similar to that obtained by Bhattacharyya [2]. Also, the classical frequency equation when both media are elastic and the other effects are absent is obtained.

2. Formulation of the problem. Consider a system of orthogonal cartesian axes $x_{1}, x_{2}, x_{3}$ such that the interface and the free surface of the granular layer resting on the granular half-space of different material are the planes $x_{3}=H$ and $x_{3}=0$, respectively, the origin $\mathrm{O}$ is any point on the free surface, $x_{3}$-axis is positive in the direction towards the exterior of the half-space, and the $x_{1}$-axis is positive along the direction of Rayleigh waves propagation.

The state of deformation in the granular medium is described by the displacement vector $\underline{\mathbf{U}}\left(u_{1}, 0, u_{3}\right)$ of the centre of gravity of a grain and the rotation vector $\underline{\xi}(\xi, \eta, \zeta)$ of the grain about its centre of gravity.

In this problem the stress tensor and the stress couple are nonsymmetric, i.e., $\boldsymbol{\tau}_{i j} \neq$ $\tau_{j i}$ and $M_{i j} \neq M_{j i}$. The stress tensor $\tau_{i j}$ can be expressed as the sum of symmetric and antisymmetric tensors

$$
\tau_{i j}=\sigma_{i j}+\sigma_{i j}^{\prime}
$$

where

$$
\sigma_{i j}=\frac{1}{2}\left(\boldsymbol{\tau}_{i j}+\boldsymbol{\tau}_{j i}\right) \quad \text { and } \quad \sigma_{i j}^{\prime}=\frac{1}{2}\left(\boldsymbol{\tau}_{i j}-\boldsymbol{\tau}_{j i}\right) .
$$

The symmetric tensor $\sigma_{i j}=\sigma_{j i}$ is related to the symmetric strain tensor

$$
e_{i j}=e_{j i}=\frac{1}{2}\left(\frac{\partial u_{i}}{\partial x_{j}}+\frac{\partial u_{j}}{\partial x_{i}}\right),
$$

by Hooke's law.

The antisymmetric stresses $\sigma_{i j}^{\prime}$ are given by

$$
\sigma_{23}^{\prime}=-F \frac{\partial \xi}{\partial t}, \quad \sigma_{31}^{\prime}=-F \frac{\partial \eta}{\partial t}, \quad \sigma_{12}^{\prime}=-F \frac{\partial \zeta}{\partial t}, \quad \sigma_{11}^{\prime}=\sigma_{22}^{\prime}=\sigma_{33}^{\prime}=0,
$$

where $F$ is the coefficient of friction.

The stress couple $M_{i j}$ is given by

$$
M_{i j}=M v_{i j}
$$

where $M$ is the third elastic constant,

$$
\begin{gathered}
v_{11}=\frac{\partial \xi}{\partial x_{1}}, \quad v_{22}=0, \quad v_{33}=\frac{\partial \zeta}{\partial x_{3}}, \quad v_{23}=0, \quad v_{31}=\frac{\partial \xi}{\partial x_{3}}, \\
v_{12}=\frac{\partial}{\partial x_{1}}\left(\omega_{2}+\eta\right), \quad v_{32}=\frac{\partial}{\partial x_{3}}\left(\omega_{2}+\eta\right), \quad v_{13}=\frac{\partial \xi}{\partial x_{3}}, \quad v_{21}=0,
\end{gathered}
$$

where $\omega_{2}=1 / 2\left(\partial u_{1} / \partial x_{3}-\partial u_{3} / \partial x_{1}\right)$ is the component of rotation.

The heat conduction equation is given by (see [6])

$$
K \nabla^{2} T=\rho C_{e} \frac{\partial T}{\partial t}+\gamma T_{0} \nabla \cdot \frac{\partial \underline{U}}{\partial t},
$$


where $K$ is the thermal conductivity, $T$ is the temperature change about the initial temperature $T_{0}, \rho$ is the density, $C_{e}$ is the specific heat per unit mass at constant strain, $\gamma$ is equal to $\alpha(3 \lambda+2 \mu), \alpha$ is the thermal expansion coefficient, and $\lambda$ and $\mu$ are Lame's constants and $t$ is the time.

The components of incremental stress in terms of the displacement are given by (see $[3,6])$

$$
\begin{gathered}
\sigma_{11}=(\lambda+2 \mu+p) \frac{\partial u_{1}}{\partial x_{1}}+(\lambda+p) \frac{\partial u_{3}}{\partial x_{3}}-\gamma T \\
\sigma_{33}=\lambda \frac{\partial u_{1}}{\partial x_{1}}+(\lambda+2 \mu) \frac{\partial u_{3}}{\partial x_{3}}-\gamma T \\
\sigma_{13}=\mu\left(\frac{\partial u_{1}}{\partial x_{3}}+\frac{\partial u_{3}}{\partial x_{1}}\right)
\end{gathered}
$$

The dynamical equations of motion are

$$
\begin{gathered}
\frac{\partial \tau_{11}}{\partial x_{1}}+\frac{\partial \tau_{13}}{\partial x_{3}}+P \frac{\partial \omega_{2}}{\partial x_{3}}=\rho \frac{\partial^{2} u_{1}}{\partial t^{2}} \\
\frac{\partial \tau_{12}}{\partial x_{1}}+\frac{\partial \tau_{32}}{\partial x_{3}}=0 \\
\frac{\partial \tau_{13}}{\partial x_{1}}+\frac{\partial \tau_{33}}{\partial x_{3}}+P \frac{\partial \omega_{2}}{\partial x_{1}}=\rho \frac{\partial^{2} u_{3}}{\partial t^{2}}
\end{gathered}
$$

and

$$
\begin{aligned}
& \tau_{23}-\tau_{32}+\frac{\partial M_{11}}{\partial x_{1}}+\frac{\partial M_{31}}{\partial x_{1}}=0 \\
& \tau_{31}-\tau_{13}+\frac{\partial M_{12}}{\partial x_{1}}+\frac{\partial M_{32}}{\partial x_{3}}=0 \\
& \tau_{12}-\tau_{21}+\frac{\partial M_{13}}{\partial x_{1}}+\frac{\partial M_{33}}{\partial x_{3}}=0 .
\end{aligned}
$$

Equations (2.9) and (2.10) take the forms, when the stresses are substituted,

$$
\begin{gathered}
(\lambda+2 \mu+P) \frac{\partial^{2} u_{1}}{\partial x_{1}^{2}}+\left(\mu+\frac{P}{2}\right) \frac{\partial^{2} u_{1}}{\partial x_{3}^{2}} \\
+\left(\lambda+\mu+\frac{P}{2}\right) \frac{\partial^{2} u_{3}}{\partial x_{1} \partial x_{3}}-\gamma \frac{\partial T}{\partial x_{1}}-F \frac{\partial}{\partial t}\left(\frac{\partial \eta}{\partial x_{3}}\right)=\rho \frac{\partial^{2} u_{1}}{\partial t^{2}} \\
\frac{\partial}{\partial t}\left(\frac{\partial \xi}{\partial x_{3}}-\frac{\partial \zeta}{\partial x_{1}}\right)=0 \\
\left(\lambda+\mu+\frac{P}{2}\right) \frac{\partial^{2} u_{1}}{\partial x_{1} \partial x_{3}}+\left(\mu-\frac{P}{2}\right) \frac{\partial^{2} u_{3}}{\partial x_{1}^{2}} \\
+(\lambda+2 \mu) \frac{\partial^{2} u_{3}}{\partial x_{3}^{2}}-\gamma \frac{\partial T}{\partial x_{3}}+F \frac{\partial}{\partial t}\left(\frac{\partial \eta}{\partial x_{1}}\right)=\rho \frac{\partial^{2} u_{3}}{\partial t^{2}} \\
-F \frac{\partial \xi}{\partial t}+M \nabla^{2} \xi=0
\end{gathered}
$$




$$
\begin{gathered}
-F \frac{\partial \eta}{\partial t}+M \nabla^{2}\left(\eta+\omega_{2}\right)=0, \\
-F \frac{\partial \zeta}{\partial t}+M \nabla^{2} \zeta=0 .
\end{gathered}
$$

3. Solution of the problem. Let the constants $\lambda, \mu, \rho, F, M, \gamma$ and $\bar{\lambda}, \bar{\mu}, \bar{\rho}, \bar{F}, \bar{M}, \bar{\gamma}$ be characteristics of the layer and the half-space, respectively. Let us introduce the displacement potentials $\phi\left(x_{1}, x_{3}, t\right)$ and $\psi\left(x_{1}, x_{3}, t\right)$ which are related to the displacement components $u_{1}$ and $u_{3}$ by the relations

$$
u_{1}=\frac{\partial \phi}{\partial x_{1}}-\frac{\partial \psi}{\partial x_{3}}, \quad u_{3}=\frac{\partial \phi}{\partial x_{3}}-\frac{\partial \psi}{\partial x_{1}} .
$$

Substituting from (3.1) into (2.11), (2.13), and (2.15), we see that $\phi$ and $\psi$ satisfy the wave equations

$$
\begin{aligned}
& \alpha^{2} \nabla^{2} \phi-\frac{\partial^{2} \phi}{\partial t^{2}}-\frac{\gamma}{\rho} T=0 \\
& \beta^{2} \nabla^{2} \psi-\frac{\partial^{2} \psi}{\partial t^{2}}+s \frac{\partial \eta}{\partial t}=0 \\
& -s^{\prime} \frac{\partial \eta}{\partial t}+\nabla^{2} \eta-\nabla^{4} \psi=0
\end{aligned}
$$

where

$$
\alpha^{2}=\frac{\lambda+2 \mu+p}{\rho}, \quad \beta^{2}=\frac{\mu-(p / 2)}{\rho}, \quad S=\frac{F}{\rho}, \quad S^{\prime}=\frac{F}{M} .
$$

From (3.1), the heat conduction equation (2.7) becomes

$$
k \nabla^{2} T=\rho C_{e} \frac{\partial T}{\partial t}+\gamma T_{0} \nabla^{2}\left(\frac{\partial \phi}{\partial t}\right) .
$$

Elimination of $T$ from (3.2) and (3.6), gives

$$
\left(\nabla^{2}-\frac{1}{\chi} \frac{\partial}{\partial t}\right)\left(\alpha^{2} \nabla^{2} \phi-\frac{\partial^{2} \phi}{\partial t^{2}}\right)-\epsilon \nabla^{2} \frac{\partial \phi}{\partial t}=0
$$

where

$$
\chi=\frac{k}{\rho c_{e}}, \quad \epsilon=\frac{\gamma^{2} T_{0}}{\rho k} .
$$

Also, $\eta$ can be eliminated by the use of equations (3.3) and (3.4) as follows:

$$
\left(\nabla^{2}-s^{\prime} \frac{\partial}{\partial t}\right)\left(\beta^{2} \nabla^{2} \psi-\frac{\partial^{2} \psi}{\partial t^{2}}\right)+S \nabla^{4} \frac{\partial \psi}{\partial t}=0 .
$$

For a plane harmonic wave propagation in the $x_{1}$-direction, we assume

$$
\begin{gathered}
\phi=\phi_{1}\left(x_{3}\right) \exp \left(i\left(L x_{1}-b t\right)\right), \\
\psi=\psi_{1}\left(x_{3}\right) \exp \left(i\left(L x_{1}-b t\right)\right), \\
(\xi, \eta, \zeta)=\left(\xi_{1}\left(x_{3}\right), \eta_{1}\left(x_{3}\right), \zeta_{1}\left(x_{3}\right)\right) \exp \left(i\left(L x_{1}-b t\right)\right),
\end{gathered}
$$

where $b$ is real positive and $L$ is in general complex. 
Substituting from (3.12) into (2.12), (2.14), and (2.16), gives

$$
\begin{aligned}
& D \xi_{1}-i L \zeta_{1}=0, \\
& D^{2} \xi_{1}+h^{2} \xi_{1}=0, \\
& D^{2} \zeta_{1}+h^{2} \zeta_{1}=0
\end{aligned}
$$

where $h^{2}=i b s^{\prime}-L^{2}, D \equiv d / d x_{3}$.

Solutions of (3.14) and (3.15) are

$$
\xi_{1}=A_{1} e^{i h x_{3}}+A_{2} e^{-i h x_{3}}, \quad \zeta_{1}=B_{1} e^{i h x_{3}}+B_{2} e^{-i h x_{3}},
$$

respectively.

From (3.13) and (3.16), we obtain

$$
h\left(A_{1} e^{i h x_{3}}-A_{2} e^{-i h x_{3}}\right)-L\left(B_{1} e^{i h x_{3}}-B_{2} e^{-i h x_{3}}\right)=0 .
$$

Equating the coefficients of $e^{i h x_{3}}$ and $e^{-i h x_{3}}$ to zero in (3.17), gives

$$
A_{1}=\frac{L}{h} B_{1}, \quad A_{2}=\frac{-L}{h} B_{2} .
$$

Also, substitution from (3.10) and (3.11) into (3.7) and (3.9), we obtain

$$
\begin{aligned}
& {\left[\alpha^{2} D^{4}+\left(b^{2}-2 L^{2} \alpha^{2}+i b \varepsilon+\frac{i b \alpha^{2}}{x}\right) D^{2}+\alpha^{2} L^{4}\right.} \\
& \left.-b^{2} L^{2}-i b L^{2} \varepsilon-\frac{i b L^{2} \alpha^{2}}{x}+\frac{i b^{3}}{x}\right] \phi_{1}=0 \\
& {\left[\left(\beta^{2}-i b s\right) D^{4}+\left(b^{2}-2 L^{2} \beta^{2}+i b s^{\prime} \beta^{2}+2 i b s L^{2}\right) D^{2}\right.} \\
& \left.+\left(\beta^{2}-i b s\right) L^{4}-\left(b+i s^{\prime} \beta^{2}\right) b L^{2}+i b^{3} S^{\prime}\right] \psi_{1}=0 .
\end{aligned}
$$

The solution of (3.19) and (3.20) has the form

$$
\begin{aligned}
& \phi_{1}=A_{3} e^{m_{3} x_{3}}+B_{3} e^{-m_{3} x_{3}}+A_{4} e^{m_{4} x_{3}}+B_{4} e^{-m_{4} x_{3}}, \\
& \psi_{1}=E_{3} e^{n_{3} x_{3}}+F_{3} e^{-n_{3} x_{3}}+E_{4} e^{n_{4} x_{3}}+F_{4} e^{-n_{4} x_{3}},
\end{aligned}
$$

where

$$
\begin{aligned}
\left(m_{3}^{2}, m_{4}^{2}\right) & =L^{2}-\frac{b(b+i \varepsilon)}{2 \alpha^{2}}-\frac{i b}{2 \chi} \pm \frac{b}{2 \alpha^{2}}\left[(b+i \varepsilon)^{2}-2 i \alpha^{2}(b+i \varepsilon)-\frac{\alpha^{4}}{\chi}\right]^{1 / 2} \\
\left(n_{3}^{2}, n_{4}^{2}\right) & =\frac{2 L^{2} \beta^{2}-b^{2}-i b \beta^{2} s^{\prime}-2 i b L^{2} s \pm b\left[\left(b-i \beta^{2} s^{\prime}\right)^{2}-4 b^{2} s s^{\prime}\right]^{1 / 2}}{2\left(\beta^{2}-i b s\right)}
\end{aligned}
$$

Using (3.3), (3.11), (3.12), and (3.22), we get

$$
\eta_{1}=\Omega_{3}\left(E_{3} e^{n_{3} x_{3}}+F_{3} e^{-n_{3} x_{3}}\right)+\Omega_{4}\left(E_{4} e^{n_{4} x_{3}}+F_{4} e^{-n_{4} x_{3}}\right),
$$


where

$$
\Omega_{3}=\frac{-i \beta^{2}}{b S}\left(n_{3}^{2}-L^{2}+\frac{b^{2}}{\beta^{2}}\right), \quad \Omega_{4}=\frac{-i \beta^{2}}{b S}\left(n_{4}^{2}-L^{2}+\frac{b^{2}}{\beta^{2}}\right) .
$$

From (3.2), (3.10), and (3.21), we have

$$
T=\left[\Omega_{3}^{\prime}\left(A_{3} e^{m_{3} x_{3}}+B_{3} e^{-m_{3} x_{3}}\right)+\Omega_{4}^{\prime}\left(A_{4} e^{m_{4} x_{3}}+B_{4} e^{-m_{4} x_{3}}\right)\right] \exp \left[i\left(L x_{1}-b t\right)\right],
$$

where

$$
\Omega_{3}^{\prime}=\frac{\rho \alpha^{2}}{\gamma}\left(m_{3}^{2}-L^{2}+\frac{b^{2}}{\alpha^{2}}\right), \quad \Omega_{4}^{\prime}=\frac{\rho \alpha^{2}}{\gamma}\left(m_{4}^{2}-L^{2}+\frac{b^{2}}{\alpha^{2}}\right) .
$$

The functions $\xi_{1}, \zeta_{1}, \eta_{1}, \phi_{1}$, and $\psi_{1}$ in the state of the lower medium must vanish as $x_{3} \rightarrow \infty$ and using the symbols with a bar for the quantities in the lower medium (except $x_{3}, L, b, p$ ) and assuming the real parts of $\bar{m}_{3}, \bar{m}_{4}, \bar{n}_{3}, \bar{n}_{4}$ are positive while the imaginary part of $\bar{h}$ is negative, we obtain, for $x_{3}>H$,

$$
\begin{aligned}
\bar{\xi}_{1} & =-\frac{L}{\bar{h}} \bar{B}_{2} e^{-i \bar{h} x_{3}}, \\
\bar{\zeta}_{1} & =\bar{B}_{2} e^{-i h x_{3}}, \\
\bar{\eta}_{1} & =\bar{\Omega}_{3} \bar{F}_{3} e^{-\bar{n}_{3} x_{3}}+\bar{\Omega}_{4} \bar{F}_{4} e^{-\bar{n}_{4} x_{3},} \\
\bar{\phi}_{1} & =\bar{B}_{3} e^{-\bar{m}_{3} x_{3}}+\bar{B}_{4} e^{-\bar{m}_{4} x_{3},} \\
\bar{\psi}_{1} & =\bar{F}_{3} e^{-\bar{n}_{3} x_{3}}+\bar{F}_{4} e^{-\bar{n}_{4} x_{3}}, \\
\bar{T} & =\bar{\Omega}_{3}^{\prime} \bar{B}_{3} e^{-\bar{m}_{3} x_{3}}+\bar{\Omega}_{4}^{\prime} \bar{B}_{4} e^{-\bar{m}_{4} x_{3}} .
\end{aligned}
$$

4. Boundary conditions and frequency equation. The boundary conditions on the interface $x_{3}=H$ are
(i) $u_{1}=\bar{u}_{1}$,
(ii) $u_{3}=\bar{u}_{3}$
(iii) $\xi=\bar{\xi}$,
(iv) $\eta=\bar{\eta}$,
(v) $\zeta=\bar{\zeta}$,
(vi) $M_{33}=\bar{M}_{33}$,
(vii) $M_{31}=\bar{M}_{31}$,
(viii) $M_{32}=\bar{M}_{32}$,
(ix) $\boldsymbol{\tau}_{33}=\overline{\boldsymbol{\tau}}_{33}$,
(x) $\tau_{31}=\bar{\tau}_{31}$,
(xi) $\tau_{32}=\bar{\tau}_{32}$,
(xii) $T=\bar{T}$,
(xiii) $\frac{\partial T}{\partial x_{3}}+\theta T=\frac{\partial \bar{T}}{\partial x_{3}}+\bar{\theta} \bar{T}$.

The boundary conditions on the free surface $x_{3}=0$ are

$$
\begin{array}{rlll}
\text { (xiv) } \quad M_{33}=0, \quad(\text { xv }) \quad M_{31}=0, \quad(\text { xvi }) \quad M_{32}=0, \quad(\text { xvii }) \quad \tau_{33}=0, \\
\text { (xviii) } \quad \tau_{31}=0, \quad(\text { xxi }) \quad \tau_{32}=0, \quad(\text { xx }) \quad \frac{\partial T}{\partial x_{3}}+\theta T=0,
\end{array}
$$

where

$$
\begin{gathered}
M_{33}=M \frac{\partial \zeta}{\partial x_{3}}, \quad M_{32}=M \frac{\partial}{\partial x_{3}}\left(\eta-\nabla^{2} \Psi\right), \quad M_{31}=M \frac{\partial \xi}{\partial x_{3}}, \\
\tau_{33}=\lambda \nabla^{2} \phi+2 \mu\left(\frac{\partial^{2} \phi}{\partial x_{3}^{2}}-\frac{\partial^{2} \psi}{\partial x_{1} \partial x_{3}}\right)-\gamma T, \quad \tau_{32}=-F \frac{\partial \xi}{\partial t}, \\
\tau_{31}=\mu\left(2 \frac{\partial^{2} \phi}{\partial x_{1} \partial x_{3}}-\frac{\partial^{2} \psi}{\partial x_{3}^{2}}+\frac{\partial^{2} \psi}{\partial x_{1}^{2}}\right)-F \frac{\partial \eta}{\partial t},
\end{gathered}
$$


$\theta$ is the ratio of the coefficients of heat transfer to the thermal conductivity.

From the boundary conditions (iii), (v), (vi), and (vii), we get

$$
\begin{aligned}
B_{1} e^{i h H}-B_{2} e^{-i h H} & =-\bar{B}_{2} e^{-i \bar{h} H}, \\
B_{1} e^{i h H}+B_{2} e^{-i h H} & =-\bar{B}_{2} e^{i \bar{h} H}, \\
M\left(B_{1} e^{i h H}-B_{2} e^{-i h H}\right) & =-\bar{M} \bar{B}_{2} e^{-i \bar{h} H}, \\
M\left(B_{1} e^{i h H}+B_{2} e^{-i h H}\right) & =-\bar{M} \bar{B}_{2} e^{-i \bar{h} H} .
\end{aligned}
$$

Whence

$$
B_{1}=B_{2}=\bar{B}_{2}=0, \quad \xi=\zeta=\bar{\xi}=\bar{\zeta}=0
$$

The other significant boundary conditions are responsible for the following relations:

$$
\begin{aligned}
& \text { (xvi) } q_{1}\left(E_{3}-F_{3}\right)+q_{2}\left(E_{4}-F_{4}\right)=0 \text {, } \\
& \text { (xvii) } q_{3}\left(A_{3}+B_{3}\right)+q_{4}\left(A_{4}+B_{4}\right)+q_{5}\left(E_{3}-F_{3}\right)+q_{6}\left(E_{4}-F_{4}\right)=0, \\
& \text { (xviii) } q_{7}\left(A_{3}-B_{3}\right)+q_{8}\left(A_{4}-B_{4}\right)+q_{9}\left(E_{3}-F_{3}\right)+q_{10}\left(E_{4}-F_{4}\right)=0 \text {, } \\
& \text { (i) } i L\left(A_{3} e^{m_{3} H}+B_{3} e^{-m_{3} H}+A_{4} e^{m_{4} H}+B_{4} e^{-m_{4} H}\right)-n_{3}\left(E_{3} e^{n_{3} H}+F_{3} e^{-n_{3} H}\right) \\
& -n_{4}\left(E_{3} e^{n_{4} H}+F_{4} e^{-n_{4} H}\right)=i L \bar{B}_{3} e^{-\bar{m}_{3} H}+i L \bar{B}_{4} e^{-\bar{m}_{4} H}+\bar{n}_{3} \bar{F}_{3} e^{-\bar{n}_{3} H}+\bar{n}_{4} \bar{F}_{4} e^{-\bar{n}_{4} H}, \\
& \text { (ii) } m_{3}\left(A_{3} e^{m_{3} H}-B_{3} e^{-m_{3} H}\right)+m_{4}\left(A_{4} e^{m_{4} H}-B_{4} e^{-m_{4} H}\right) \\
& +i L\left(E_{3} e^{n_{3} H}-F_{3} e^{-n_{3} H}+E_{4} e^{n_{4} H}+F_{4} e^{-n_{4} H}\right) \\
& =-\bar{m}_{3} \bar{B}_{3} e^{-\bar{m}_{3} H}-\bar{m}_{4} \bar{B}_{4} e^{-\bar{m}_{4} H}+i L \bar{F}_{3} e^{-\bar{n}_{3} H}+i L \bar{F}_{4} e^{-\bar{n}_{4} H}, \\
& =-\bar{M}\left(\bar{q}_{1} \bar{F}_{3} e^{-\bar{n}_{3} H}+\bar{q}_{2} \bar{F}_{4} e^{-\bar{n}_{4} H}\right), \\
& +q_{6}\left(E_{4} e^{n_{4} H}-F_{4} e^{-n_{4} H}\right)=\bar{q}_{3} \bar{B}_{3} e^{-\bar{m}_{3} H}+\bar{q}_{4} \bar{B}_{4} e^{-\bar{m}_{4} H}-\bar{q}_{5} \bar{F}_{3} e^{-\bar{n}_{3} H}-\bar{q}_{6} \bar{F}_{4} e^{-\bar{n}_{4} H}, \\
& +q_{10}\left(E_{4} e^{n_{4} H}-F_{4} e^{-n_{4} H}\right)=-\bar{q}_{7} \bar{B}_{3} e^{-\bar{m}_{3} H}-\bar{q}_{8} \bar{B}_{4} e^{-\bar{m}_{4} H} \\
& -\bar{q}_{9} \bar{F}_{3} e^{-\bar{n}_{3} H}-\bar{q}_{10} \bar{F}_{4} e^{-\bar{n}_{4} H},
\end{aligned}
$$




$$
\text { (xiii) } \begin{aligned}
q_{11} A_{3} e^{m_{3} H}+q_{12} B_{3} e^{-m_{3} H} & +q_{13} A_{4} e^{m_{4} H}+q_{14} B_{4} e^{-m_{4} H} \\
& =\bar{q}_{12} \bar{B}_{3} e^{-\bar{m}_{3} H}+\bar{q}_{14} \bar{B}_{4} e^{-\bar{m}_{4} H},
\end{aligned}
$$$$
\text { (xx) } q_{11} A_{3}+q_{12} B_{3}+q_{13} A_{4}+q_{14} B_{4}=0
$$

where

$$
\begin{array}{rlrl}
q_{1} & =n_{3}\left(\Omega_{3}+L^{2}-n_{3}^{2}\right), & & \bar{q}_{1}=\bar{n}_{3}\left(\bar{\Omega}_{3}+L^{2}-\bar{n}_{3}^{2}\right), \\
q_{2} & =n_{4}\left(\Omega_{4}+L^{2}-n_{4}^{2}\right), & & \bar{q}_{2}=\bar{n}_{4}\left(\bar{\Omega}_{4}+L^{2}-\bar{n}_{4}^{2}\right), \\
q_{3} & =(2 \mu+p) L^{2}-\rho b^{2}-p m_{3}^{2},, & & \bar{q}_{3}=(2 \bar{\mu}+p) L^{2}-\bar{\rho} b^{2}-p \bar{m}_{3}^{2}, \\
q_{4} & =(2 \mu+p) L^{2}-\rho b^{2}-p m_{4}^{2}, & & \bar{q}_{4}=(2 \bar{\mu}+p) L^{2}-\bar{\rho} b^{2}-p \bar{m}_{4}^{2}, \\
q_{5} & =-2 i L \mu n_{3}, & \bar{q}_{5} & =-2 i L \bar{\mu} \bar{n}_{3}, \\
q_{6} & =-2 i L \mu n_{4}, & \bar{q}_{6} & =2 i L \bar{\mu} \bar{n}_{4}, \\
q_{7} & =2 i L \mu m_{3}, & \bar{q}_{7} & =2 i L \bar{\mu} \bar{m}_{3}, \\
q_{8} & =2 i L \mu m_{4}, & \bar{q}_{8}=2 i L \bar{\mu} \bar{m}_{4}, \\
q_{9} & =i b F \Omega_{3}-\mu L^{2}-\mu n_{3}^{2}, & \bar{q}_{9}=i b \bar{F} \bar{\Omega}_{3}-\bar{\mu}^{2}-\bar{\mu} \bar{n}_{3}^{2}, \\
q_{10} & =i b F \Omega_{4}-\mu L^{2}-\mu n_{4}^{2}, & \bar{q}_{10} & =i b \bar{F} \bar{\Omega}_{4}-\bar{\mu} L^{2}-\bar{\mu} \bar{n}_{4}^{2}, \\
q_{11} & =\Omega_{3}^{\prime}\left(\theta+m_{3}\right), & \bar{q}_{11} & =\bar{\Omega}_{3}^{\prime}\left(\bar{\theta}+\bar{m}_{3}\right), \\
q_{12} & =\Omega_{3}^{\prime}\left(\theta-m_{3}\right), & \bar{q}_{12} & =\bar{\Omega}_{3}^{\prime}\left(\bar{\theta}-\bar{m}_{3}\right), \\
q_{13} & =\Omega_{4}^{\prime}\left(\theta+m_{4}\right), & \bar{q}_{13} & =\bar{\Omega}_{4}^{\prime}\left(\bar{\theta}+\bar{m}_{4}\right), \\
q_{14} & =\Omega_{4}^{\prime}\left(\theta-m_{4}\right), & \bar{q}_{14} & =\bar{\Omega}_{4}^{\prime}\left(\bar{\theta}-\bar{m}_{4}\right),
\end{array}
$$

Elimination of $A_{3}, B_{3}, A_{4}, B_{4}, E_{3}, F_{3}, E_{4}, F_{4}, \bar{B}_{3}, \bar{B}_{4}, \bar{F}_{3}, \bar{F}_{4}$ gives the wave velocity equation in the form of

$$
\operatorname{det} d_{i j}=0 \text {, }
$$

where the non-vanishing entries of the twelfth-order determinant of $d_{i j}$ are given by

$$
\begin{array}{llll}
d_{15}=q_{1} e^{-n_{3} H}, & d_{16}=-q_{1} e^{n_{3} H}, & d_{17}=q_{2} e^{-n_{4} H}, & d_{18}=-q_{2} e^{n_{4} H}, \\
d_{21}=q_{3} e^{-m_{3} H}, & d_{22}=q_{3} e^{m_{3} H}, & d_{23}=q_{4} e^{-m_{4} H}, & d_{24}=q_{4} e^{m_{4} H}, \\
d_{25}=q_{5} e^{-n_{3} H}, & d_{26}=-q_{5} e^{n_{3} H}, & d_{27}=q_{6} e^{-n_{4} H}, & d_{28}=-q_{6} e^{n_{4} H}, \\
d_{31}=q_{7} e^{-m_{3} H}, & d_{32}=-q_{7} e^{m_{3} H}, & d_{33}=q_{8} e^{-m_{4} H}, & d_{34}=-q_{8} e^{m_{4} H}, \\
d_{35}=q_{9} e^{-n_{3} H}, & d_{36}=-q_{9} e^{n_{3} H}, & d_{37}=q_{10} e^{-n_{4} H}, & d_{38}=-q_{10} e^{n_{4} H}, \\
d_{41}=i L, & d_{42}=i L, & d_{43}=i L, & d_{44}=i L, \\
d_{45}=-n_{3}, & d_{46}=-n_{3}, & d_{47}=-n_{4}, & d_{48}=n_{4}, \\
d_{49}=-i L, & d_{410}=i L, & d_{411}=-\bar{n}_{3}, & d_{412}=-\bar{n}_{4}, \\
d_{51}=m_{3}, & d_{52}=-m_{3}, & d_{53}=m_{4}, & d_{54}=-m_{4}, \\
d_{55}=i L, & d_{56}=i L, & d_{57}=i L, & d_{58}=i L, \\
d_{59}=\bar{m}_{3}, & d_{510}=\bar{m}_{4}, & d_{511}=i L, & d_{512}=i L, \\
d_{65}=\Omega_{3}, & d_{66}=\Omega_{3}, & d_{67}=\Omega_{4}, & d_{68}=\Omega_{4},
\end{array}
$$




$\begin{array}{llll}d_{611}=-\bar{\Omega}_{3}, & d_{612}=-\bar{\Omega}_{4}, & d_{75}=M q_{1}, & d_{76}=-M q_{1}, \\ d_{77}=M q_{2}, & d_{78}=-M q_{2}, & d_{711}=\bar{M} \bar{q}_{1}, & d_{712}=-\bar{M} \bar{q}_{2}, \\ d_{81}=q_{3}, & d_{82}=q_{3}, & d_{83}=q_{4}, & d_{84}=q_{4}, \\ d_{85}=q_{5}, & d_{86}=-q_{5}, & d_{87}=q_{6}, & d_{88}=-q_{6}, \\ d_{89}=-\bar{q}_{3}, & d_{810}=-\bar{q}_{4}, & d_{811}=\bar{q}_{5}, & d_{812}=\bar{q}_{6}, \\ d_{91}=q_{7}, & d_{92}=-q_{7}, & d_{93}=q_{8}, & d_{94}=-q_{8}, \\ d_{95}=q_{9}, & d_{96}=-q_{9}, & d_{97}=q_{10}, & d_{98}=-q_{10}, \\ d_{99}=\bar{q}_{7}, & d_{910}=\bar{q}_{8}, & d_{911}=\bar{q}_{9}, & d_{912}=\bar{q}_{10}, \\ d_{101}=\Omega_{3}^{\prime}, & d_{102}=\Omega_{3}^{\prime}, & d_{103}=\Omega_{4}^{\prime}, & d_{104}=\Omega_{4}^{\prime}, \\ d_{109}=-\bar{\Omega}_{3}^{\prime}, & d_{1010}=-\bar{\Omega}_{4}^{\prime}, & d_{111}=q_{11}, & d_{112}=q_{12}, \\ d_{113}=q_{13}, & d_{114}=q_{14}, & d_{119}=-\bar{q}_{12}, & d_{1110}=-\bar{q}_{14}, \\ d_{121}=q_{11} e^{-m_{3} H}, & d_{122}=q_{12} e^{m_{3} H}, & d_{123}=q_{13} e^{-m_{4} H}, & d_{124}=q_{14} e^{m_{4} H} .\end{array}$

Equation (4.7) determines the wave velocity equation for the Rayleigh waves in a thermoelastic granular medium under initial stress.

5. Discussion. The transcendental equation (4.7), in the determinant form, has complex roots. The real part gives the velocity of Rayleigh waves and the imaginary part gives the attenuation due to the granular nature of the medium. It is clear from the frequency equation (4.7) that the phase velocity depends on the initial stress $P$, the friction $F$, and the coupling factor $\epsilon$.

When there is no coupling between the temperature and strain fields, we have $\theta$ vanishes,

$$
\lim _{\epsilon \rightarrow 0}\left(m_{3}^{2}, m_{4}^{2}\right)=\left(L^{2}-\frac{b^{2}}{\alpha^{2}}, L^{2}\right), \quad \lim _{\gamma \rightarrow 0}\left(\gamma \cdot \Omega_{3}^{\prime}\right)=0, \quad \lim _{\gamma \rightarrow 0}\left(\gamma \cdot \Omega_{4}^{\prime}\right)=b^{2},
$$

where

$$
\lim _{\gamma \rightarrow 0} q_{11}=0, \quad \lim _{\gamma \rightarrow 0} q_{12}=0, \quad \lim _{\gamma \rightarrow 0} q_{13}=b^{2} L, \quad \lim _{\gamma \rightarrow 0} q_{14}=-b^{2} L .
$$

Similar results hold for the lower medium. Multiplying the rows 10, 11 and 12 of the determinant $\left|d_{i j}\right|$ by $\gamma$ and then taking $\lim _{\gamma \rightarrow 0}$, equation (4.7) reduces, after some computation, to the following ninth-order determinantal equation:

$$
\left|\begin{array}{ccccccccc}
0 & 0 & q_{1} e^{-n_{3} H} & -q_{1} e^{n_{3} H} & q_{2} e^{-n_{4} H} & -q_{2} e^{n_{4} H} & 0 & 0 & 0 \\
q_{3} e^{-m_{3} H} & q_{3} e^{m_{3} H} & q_{5} e^{-n_{3} H} & -q_{5} e^{n_{3} H} & q_{6} e^{-n_{4} H} & -q_{6} e^{n_{4} H} & 0 & 0 & 0 \\
q_{7} e^{-m_{3} H} & -q_{7} e^{m_{3} H} & q_{9} e^{-n_{3} H} & -q_{9} e^{n_{3} H} & q_{10} e^{-n_{4} H} & -q_{10} e^{n_{4} H} & 0 & 0 & 0 \\
i L & i L & -n_{3} & -n_{3} & -n_{4} & n_{4} & -i L & -\bar{n}_{3} & -\bar{n}_{4} \\
m_{3} & -m_{3} & i L & i L & i L & i L & \bar{m}_{3} & -i L & -i L \\
0 & 0 & \Omega_{3} & \Omega_{3} & \Omega_{4} & \Omega_{4} & 0 & -\bar{\Omega}_{3} & -\bar{\Omega}_{4} \\
0 & 0 & M q_{1} & -M q_{1} & M q_{2} & -M q_{2} & 0 & \bar{M}_{1} \bar{q}_{1} & \bar{M} \bar{q}_{2} \\
q_{3} & q_{3} & q_{5} & -q_{5} & q_{6} & -q_{6} & -\bar{q}_{3} & \bar{q}_{5} & \bar{q}_{6} \\
q_{7} & -q_{7} & q_{9} & -q_{9} & q_{10} & -q_{10} & \bar{q}_{7} & \bar{q}_{9} & \bar{q}_{10}
\end{array}\right|=0 \text {, }
$$


where

$$
\begin{aligned}
q_{1} & =n_{3}\left(\Omega_{3}+L^{2}-n_{3}^{2}\right), & & \bar{q}_{1}=\bar{n}_{3}\left(\bar{\Omega}_{3}+L^{2}-\bar{n}_{3}^{2}\right), \\
q_{2} & =n_{4}\left(\Omega_{4}+L^{2}-n_{4}^{2}\right), & & \bar{q}_{2}=\bar{n}_{4}\left(\bar{\Omega}_{4}+L^{2}-\bar{n}_{4}^{2}\right), \\
q_{3} & =2 \mu L^{2}-b^{2}\left(\rho-\frac{P}{\alpha^{2}}\right), & & \bar{q}_{3}=2 \bar{\mu} L^{2}-b^{2}\left(\bar{\rho}-\frac{P}{\bar{\alpha}^{2}}\right), \\
q_{4} & =2 \mu L^{2}-\rho b^{2}, & \bar{q}_{4} & =2 \bar{\mu} L^{2}-\bar{\rho} b^{2}, \\
q_{5} & =-2 i L \mu n_{3}, & \bar{q}_{5} & =-2 i L \bar{\mu} \bar{n}_{3}, \\
q_{6} & =-2 i L \mu n_{4}, & \bar{q}_{6} & =-2 i L \bar{\mu} \bar{n}_{4}, \\
q_{7} & =2 i L \mu m_{3}, & \bar{q}_{7} & =2 i L \bar{\mu} \bar{m}_{3}, \\
q_{9} & =i b F \Omega_{3}-\mu L^{2}-\mu n_{3}^{2}, & \bar{q}_{9} & =i b \bar{F} \bar{\Omega}_{3}-\bar{\mu} L^{2}-\bar{\mu} \bar{n}_{3}^{2}, \\
q_{10} & =i b F \Omega_{4}-\mu L^{2}-\mu n_{4}^{2}, & \bar{q}_{10} & =i b \bar{F} \bar{\Omega}_{4}-\bar{\mu} L^{2}-\bar{\mu} \bar{n}_{4}^{2} .
\end{aligned}
$$

The frequency equation (5.3) determines the wave velocity equation for the Rayleigh waves in a granular medium under initial stress.

When the initial stress is absent, we have

$$
\alpha^{2}=\frac{\lambda+2 \mu}{\rho}, \quad B^{2}=\frac{\mu}{\rho}, \quad q_{3}=2 \mu L^{2}-\rho b^{2}, \quad \bar{q}_{3}=2 \bar{\mu} L^{2}-\bar{\rho} b^{2} .
$$

Thus, equation (5.3) with the relations (5.5) reduces to the frequency equation obtained by Bhattacharyya [2].

If the granular rotations vanish, we get

$$
\begin{gathered}
\lim _{M \rightarrow 0} \lim _{S \rightarrow 0}\left(n_{3}^{2}, n_{4}^{2}\right)=\left(L^{2}, L^{2}-\frac{b^{2}}{\beta^{2}}\right), \quad \lim _{M \rightarrow 0} \lim _{S \rightarrow 0}\left(S \cdot \Omega_{3}\right)=-i b, \\
\lim _{M \rightarrow 0} \lim _{S \rightarrow 0}\left(S \cdot \Omega_{4}\right)=0, \quad \lim _{M \rightarrow 0} \lim _{S \rightarrow 0}\left(\Omega_{4}\right)=-\frac{b^{2}}{\beta^{2}} \\
\lim _{M \rightarrow 0} \lim _{S \rightarrow 0} q_{9}=\rho b^{2}-2 \mu L^{2}, \quad \lim _{M \rightarrow 0} \lim _{S \rightarrow 0} q_{10}=-\mu\left(2 L^{2}-\frac{b^{2}}{\beta^{2}}\right) .
\end{gathered}
$$

Similar results also hold for the lower medium. Multiplying the columns 5, 6 and 11 of the determinant $\left|d_{i j}\right|$ by $S$ and then taking $\lim _{M \rightarrow 0} \lim _{S \rightarrow 0}$, we get after some computation, the following ninth-order determinantal equation:

$\mid \begin{array}{ccccccccc}q_{3} e^{-m_{3} H} & q_{3} e^{m_{3} H} & q_{4} e^{-m_{4} H} & q_{4} e^{m_{4} H} & q_{6} e^{-n_{4} H} & -q_{6} e^{n_{4} H} & 0 & 0 & 0 \\ q_{7} e^{-m_{3} H} & q_{7} e^{m_{3} H} & q_{8} e^{-m_{4} H} & -q_{8} e^{m_{4} H} & q_{10} e^{-n_{4} H} & q_{10} e^{n_{4} H} & 0 & 0 & 0 \\ i L & i L & i L & i L & -n_{4} & n_{4} & -i L & -i L & -\bar{n}_{4} \\ m_{3} & -m_{3} & m_{4} & -m_{4} & i L & i L & \bar{m}_{3} & \bar{m}_{4} & -i L \\ q_{3} & q_{3} & q_{4} & q_{4} & -q_{6} & q_{6} & -\bar{q}_{3} & -\bar{q}_{4} & \bar{q}_{6} \\ q_{7} & -q_{7} & q_{8} & -q_{8} & -q_{10} & q_{10} & \bar{q}_{7} & \bar{q}_{8} & \bar{q}_{10} \\ \Omega_{3}^{\prime} & \Omega_{3}^{\prime} & \Omega_{4}^{\prime} & -\Omega_{4}^{\prime} & 0 & 0 & -\bar{\Omega}_{3}^{\prime} & -\bar{\Omega}_{4}^{\prime} & 0 \\ q_{11} & q_{12} & q_{13} & q_{14} & 0 & 0 & \bar{q}_{12} & -\bar{q}_{14} & 0 \\ q_{11} e^{-m_{3} H} & q_{12} e^{m_{3} H} & q_{13} e^{-m_{4} H} & q_{14} e^{m_{4} H} & 0 & 0 & 0 & 0 & 0\end{array}$


Equation (5.7) is the velocity equation of an initially stressed thermoelastic granular layer of thickness $H$ overlaying semi-infinite elastic isotropic medium.

Finally, in the absence of initial stress and when there is no coupling between the temperature and strain fields, as well as the vanishing of granular rotations, equation (5.7) takes the form

$\left|\begin{array}{cccccc}R^{2} e^{m_{3} H} & 2 L m_{4} e^{m_{4} H} & R^{2} e^{-m_{3} H} & -2 L m_{4} e^{-m_{4} H} & 0 & 0 \\ 2 L m_{3} e^{m_{3} H} & R^{2} e^{m_{4} H} & -2 L m_{3} e^{-m_{3} H} & R^{2} e^{-m_{4} H} & 0 & 0 \\ -L & -m_{4} & -L & m_{4} & L & -\bar{m}_{4} \\ -m_{3} & -L & m_{3} & -L & -\bar{m}_{3} & L \\ 2 L m_{3} & R^{2} & -2 L m_{3} & R^{2} & -2 L \frac{\mu}{\mu} \bar{m}_{3} & \frac{\bar{\mu}}{\mu} \bar{R}^{2} \\ R^{2} & 2 L m_{4} & R^{2} & -2 L \bar{m}_{4} & -\frac{\bar{\mu}}{\mu} \bar{R}^{2} & -2 L \frac{\mu}{\mu} \bar{m}_{4}\end{array}\right|=0$,

where

$$
\begin{gathered}
m_{3}^{2}=L^{2}-\frac{\rho b^{2}}{\lambda+2 \mu}, \quad \bar{m}_{3}^{2}=L^{2}-\frac{\bar{\rho} b^{2}}{\bar{\lambda}+b \bar{\mu}}, \\
\beta^{2}=\frac{\mu}{\rho}, \quad \bar{\beta}^{2}=\frac{\bar{\mu}}{\bar{\rho}}, \\
m_{4}^{2}=L^{2}-\frac{b^{2}}{\beta^{2}}, \quad \bar{m}_{4}^{2}=L^{2}-\frac{b^{2}}{\bar{\beta}^{2}}, \quad R^{2}=\left(2 L^{2}-\frac{b^{2}}{\beta^{2}}\right), \quad \bar{R}^{2}=\left(2 L^{2}-\frac{b^{2}}{\bar{\beta}^{2}}\right)
\end{gathered}
$$

Equation (5.8) is identical to [5, equation (4.195)].

\section{REFERENCES}

[1] S. M. Ahmed, Influence of gravity on the propagation of waves in granular medium, Appl. Math. Comput. 101 (1999), 269-280.

[2] R. K. Bhattacharyya, Rayleigh waves in granular medium, Pure Appl. Geophys. 62 (1965), no. 3, 13-22.

[3] M. A. Biot, Mechanics of Incremental Deformations. Theory of elasticity and viscoelasticity of initially stressed solids and fluids, including thermodynamic foundations and applications to finite strain, John Wiley \& Sons Inc., New York, 1965. MR 32\#3333.

[4] A. M. Elnaggar, On the dynamical problem of a generalized thermoelastic granular infinite cylinder under initial stress, Astrophys. Space Sci. 190 (1992), no. 2, 177-190. MR 93d:73009. Zbl 755.73039.

[5] W. M. Ewing, W. S. Jardetzky, and F. Press, Elastic Waves in Layered Media, McGraw-Hill Book Co., Inc., New York, 1957. MR 20\#1475. Zbl 083.23705.

[6] W. Nowacki, Thermoelasticity, Addison-Wesely Publishing Company, Inc. London, 1962.

[7] N. Oshima (ed.), Proc. 3rd. Japan Nat. Congr. Appl. Mech., vol. 77, 1954.

[8] _ Mem. Unifying Study of Basic Problems in Engineering Sciences by Means of Geometry, vol. I. III, General Editor, Kondo, K., 1955.

[9] G. Paria, Bending of a shallow spherical shell under uniform pressures with the boundary partly clamped and partly simply-supported, Bull. Calcutta Math. Soc. 52 (1960), no. 195, 79-86. MR 23\#B2648. Zbl 098.16203. 


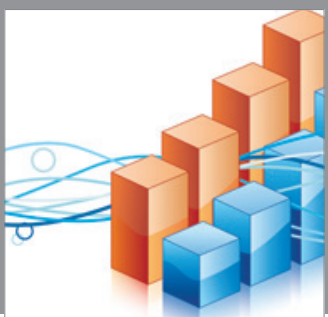

Advances in

Operations Research

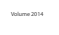

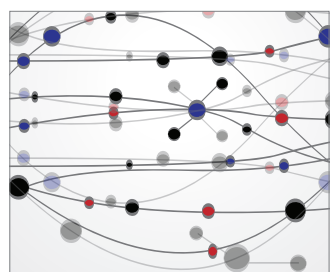

\section{The Scientific} World Journal
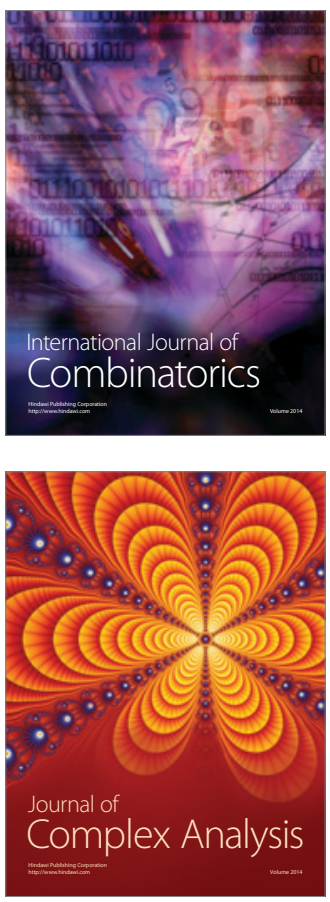

International Journal of

Mathematics and

Mathematical

Sciences
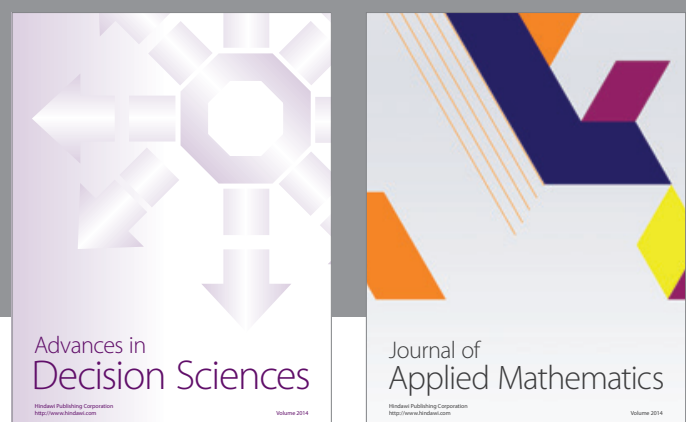

Journal of

Applied Mathematics
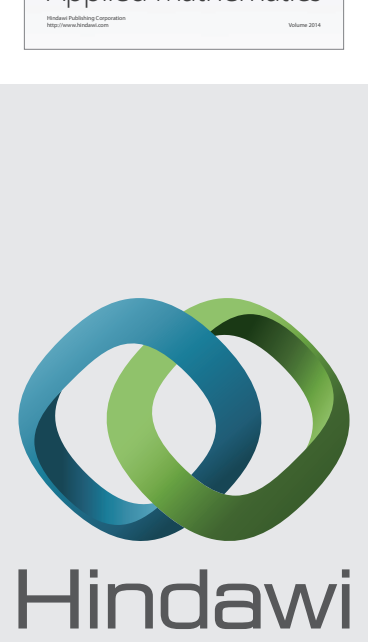

Submit your manuscripts at http://www.hindawi.com
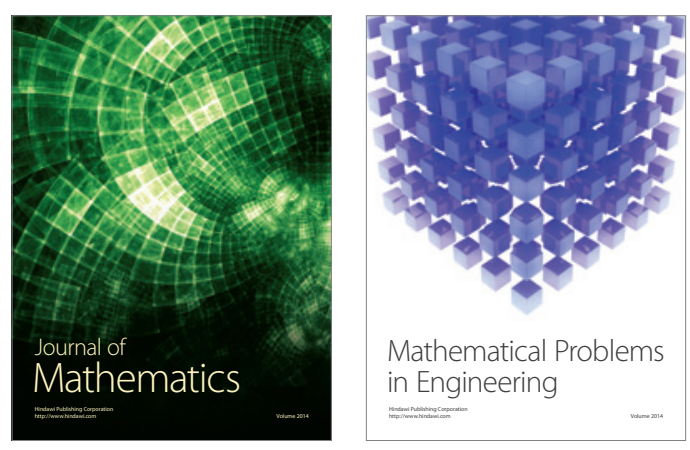

Mathematical Problems in Engineering
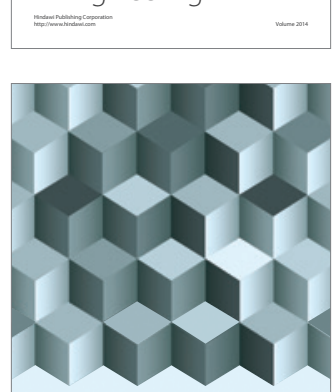

Journal of

Function Spaces
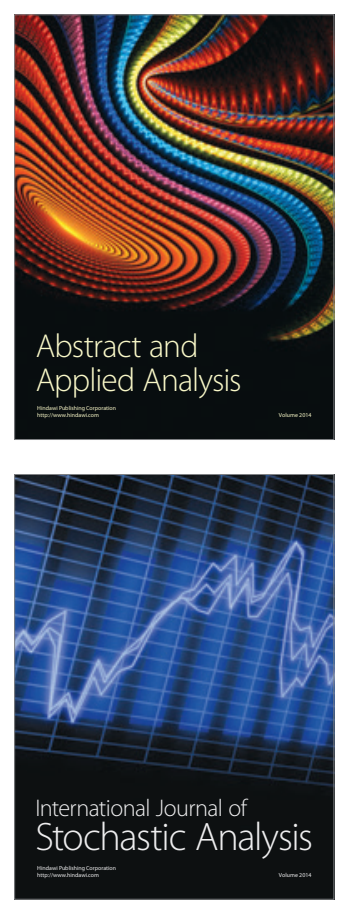

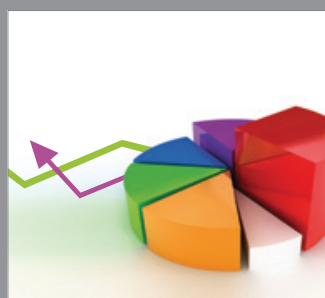

ournal of

Probability and Statistics

Promensencen
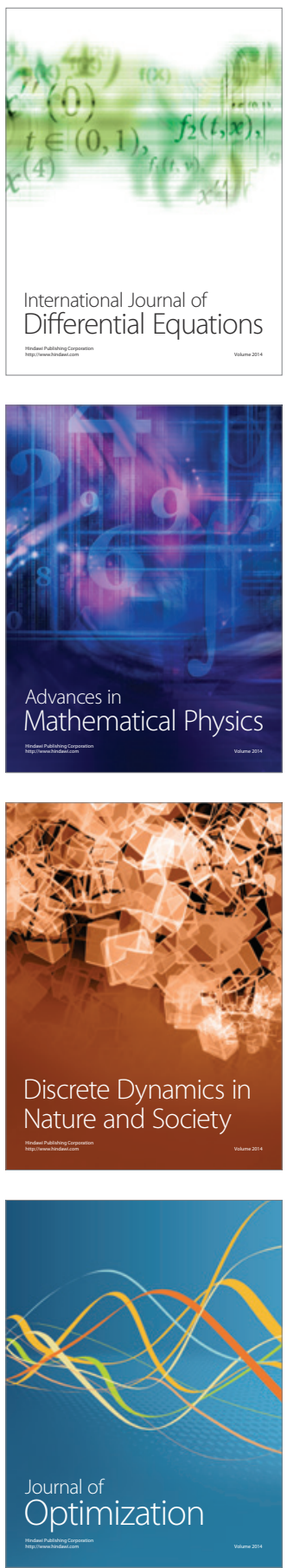